Número 53 / Septiembre 2015

\title{
LAS REDES SOCIALES: EL ACOMPAÑAMIENTO EMOCIONAL DE LOS ESTUDIANTES DURANTE LA MOVILIDAD ESTUDIANTIL UNIVERSITARIA
}

\author{
SOCIAL NETWORKS, THE EMOTIONAL STUDENTS SUPPORT DURING \\ UNIVERSITY STUDENTS MOBILITY
}

María Alejandra Rocha; arocha@ucol.mx

Genoveva Amador; genoveva@ucol.mx

Universidad de Colima

Ibis Marlene Alvarez; ibismarlene.alvarez@uab.es

Universitat Autónoma de Barcelona

\section{RESUMEN}

Esta investigación exploró el papel de las redes sociales en las experiencias de movilidad académica reportadas por 114 estudiantes de la Universidad de Colima (México). Los datos fueron recopilados mediante un cuestionario y una entrevista focal. Además, con el consentimiento de algunos participantes se analizó el contenido de los muros de los grupos de Facebook - los resultados mostraron que fue la red social más utilizada- en los que compartieron tales experiencias. El uso de las redes sociales tuvo un impacto emocional positivo y ayudó a satisfacer importantes necesidades de comunicación con amigos y familiares. Este hecho se asoció de manera significativa con la valoración de la utilidad de la movilidad estudiantil en el plano socio-afectivo. 
PALABRAS CLAVE: redes sociales, movilidad académica, educación superior, TIC, comunicación.

\section{ABSTRACT}

This research explored the role of social networks in Academic Mobility experiences reported by 114 students of the University of Colima (Mexico). Data were collected through a questionnaire and a focal interview. With the consent of some participants the content of facebook walls -results showed facebook as the most used social network -, in which they shared their experiences, was analized. The use of social networks had a positive emotional impact and helped students to satisfy important needs of communication with friends and family. This was significantly associated with the appreciation of the usefulness of student mobility in the socio-affective level.

KEY WORDS: social networks, academic mobility, higher education, ICT, communication

\section{INTRODUCCIÓN}

Actualmente, crece el interés y las oportunidades en relación a la movilidad académica en el ámbito universitario (Brovetto, 2008; Haug, 2010). Algunos alumnos se sienten "empujados" a estudiar en otros países porque en los suyos carecen de las mismas oportunidades, y otros quizá lo hagan porque desean ampliar su horizonte cultural e intelectual. En cuanto a la repercusión de Internet sobre la movilidad estudiantil se sabe poco, en tanto es difícil evaluar sus repercusiones, pero cabe suponer que ejercen un efecto sobre las tendencias en materia de movilidad estudiantil (UNESCO, 2009).

El proyecto de investigación el "Papel de las redes sociales por internet en el ajuste sociocultural en las comunidades receptoras de los estudiantes de movilidad de la Universidad de Colima", se orientó precisamente a explorar las posibilidades que ofrecen las redes sociales para la integración de los estudiantes universitarios que participan en experiencias de movilidad académica. El estudio que aquí se presenta está ligado a esta investigación.

Los estudiantes universitarios se entusiasman con la idea de la movilidad al margen de la oficialidad y de la formalidad que requieren estas decisiones especialmente en el ámbito universitario, por lo que es fácil encontrar una red social que brinde asistencia diversa en este sentido (Castells, 2004). Sin embargo, no todos los estudiantes utilizan las oportunidades que brindan al respecto las redes sociales ni las instituciones académicas 
aprovechan este recurso, sino que se dejan en manos de la comunidad estudiantil. Esto no siempre resulta en experiencias exitosas, lo cual repercute de modo desfavorable en el proceso de transición transcultural que tiene lugar cuando arriban a los países receptores (Coles y Swami, 2012).

El éxito de la red social está relacionado con su simplicidad y con la concentración de herramientas diversas para publicar. Con frecuencia se convierte en un espacio donde prima la "viralidad", la superficialidad y la fugacidad de contenidos, donde todo es intuitivo y donde se promueve una experiencia más bien lúdica. No obstante, el extenso uso que los jóvenes hacen de ella conduce a estudiar los contenidos y los formatos de comunicación que le dan vida. Se puede averiguar, por ejemplo, las oportunidades que ofrecen estos espacios, no sólo como medio de comunicación, sino como otra manera de ver y de participar en una sociedad cada vez más interconectada (Rodera, 2011).

Varios estudios corroboran que las redes sociales han captado el interés de muchos adolescentes y adultos jóvenes. Informes recientes sobre la prevalencia de las actividades en línea indican que la mayoría de los adolescentes de Estados Unidos y de los adultos jóvenes utilizan los sitios de redes sociales (Lenhart y Madden, 2007). Una investigación en la cual participaron estudiantes de distintas universidades en el Medio Oeste de Estados Unidos, encontró que el $91 \%$ de los encuestados reportó la utilización de Facebook para apoyar sus actividades de estudio (Miller, Parsons y Lifer, 2010).

"Pasar el tiempo" en las redes sociales parece ser parte de las actividades diarias de la mayoría de los jóvenes adultos. En el conjunto de medios de comunicación a través de Internet, las redes sociales se encuentran en el punto más alto en los procesos comunicativos, sobre todo en el segmento de la población juvenil (Martínez y Lazo, 2011). El estudio realizado por Ellison, Steinfield y Lampe (2007), encontró que los estudiantes universitarios de Estados Unidos utilizaban Facebook entre 10 y 30 minutos diarios. Según la Asociación para la Investigación de los Medios de Comunicación (AIMC), un 83,90\% de los internautas usan redes sociales y de ellos el 89,90\% están en Facebook; los datos del buscador Alexa.com sitúan este porcentaje en el 41,36\%" (citado en Túñez y García, 2012). Según informe de la Asociación Mexicana de Internet (AMIPICI), en México la tendencia de acceso al Internet aumentó un 13\% sólo entre 2012 y 2013. El mayor porcentaje de usuarios se encuentra entre los 13 y los 18 años (24\%) seguido por el de 19 a 24 años con un $21 \%$. El tiempo estimado de conexión es de 5 horas y 36 minutos, 9 de cada 10 acceden a una red social y la red de referencia es mayoritariamente Facebook, seguida de YouTube, Twitter y Google+.

\subsection{Oportunidades educativas de las redes sociales}


En correspondencia con la finalidad de nuestra investigación buscamos explicar el alcance y el impacto de los procesos comunicativos que tienen lugar a través de las redes sociales. En específico, prestamos atención a la funcionalidad de las redes sociales en términos de relaciones entre diferentes individuos; las cuales son generadas a partir de dos situaciones, principalmente: el conocimiento en común o el hecho de compartir gustos, lugares, intereses (Boyd y Ellison, 2007).

Las redes sociales, tal como ahora las conocemos, son sitios que promueven la interacción social de los participantes. Técnicamente los sitios de redes sociales (SNS; social network sites) pueden definirse como asociaciones de personas ligadas por motivos heterogéneos y que conforman una estructura compuesta por nodos unidos entre ellos por más de un tipo de relación (Boyd y Ellison, 2007). En los últimos años las redes sociales han experimentado un rápido aumento de popularidad y han generado oportunidades para mejorar el aprendizaje (fomentan el diálogo entre compañeros, promueven compartir recursos, facilitan la colaboración y desarrollan destrezas de comunicación) y también preocupaciones (privacidad, autoría del contenido).

Los integrantes de una red tienen objetivos de aprendizajes comunes, se esfuerzan por crear un terreno común para compartir sus experiencias en las clases. Como resultado de estos intercambios se crean lazos interpersonales, conocimiento común; en definitiva, se crea una comunidad (Haythornthwaite, 2002).

Sin embargo, sabemos muy poco acerca de cómo este fenómeno impacta en la experiencia de los estudiantes y, en particular, cómo influye en su aprendizaje. Más bien parece ser que se utiliza de manera informal, sobre todo para hablar y hablar con los amigos sobre el trabajo que para realmente hacer el trabajo (Madgea, Meekb, Wellensc y Hooley, 2009).

Las emociones son sociales y colectivas tanto por su génesis como por los procesos de transformación y reajuste que experimentan a lo largo de una vida. Incluso, antes del advenimiento del mundo virtual, las emociones eran ya emociones-en-red. "Sin ellas, la construcción de la relación entre nuevos usuarios y nuevas identidades carecería de un componente fundamental de las prácticas sociales entre unos nuevos usuarios y una sociedad digital de acogida" (Belli y De Eugenio, 2014: 59).

Este propósito no es difícil de conseguir, en tanto las redes se definen por atributos de autonomía, resistencia reducida al flujo de información, facilidad de conexión, crecimiento orgánico, iteración rápida y mejora de ideas y conceptos, además de facilidad de escalabilidad (Arnold y Paulus, 2010). La situación actual es realmente un desafío: los SNS 
más populares son los de carácter general, como Facebook, MySpace y Twitter (Álvarez y Olivera-Smith, 2013).

Varias investigaciones han demostrado que el sentido de comunidad en los entornos de aprendizaje en línea contribuye a la motivación del estudiante y repercute positivamente en su rendimiento académico (Ertmer y Stepich, 2004; Palloff y Pratt, 1999; Wilson, 2001).

Es importante atender el creciente número de estudiantes interesados en estas oportunidades, pues cada vez son más los que realizan esta experiencia no sólo porque lo permiten sus programas educativos, sino porque las instituciones de educación superior los incentivan, en aras de facilitar los llamados procesos de internacionalización de la Educación Superior (De Wit, 2011). En 2012, a pocos años de haberse instaurado los programas de movilidad estudiantil, se reportó la participación de más de cinco millones de estudiantes internacionales en el mundo, cifra superior a las predicciones hechas por la Organización para la Cooperación y el Desarrollo Económico (OCDE) para este año. No obstante el incremento significativo, esta cifra apenas representa el $2.7 \%$ de la matrícula en educación superior en el mundo (Marmolejo, 2012). En México, concretamente en la Universidad de Colima, un balance realizado al respecto también mostró un bajo indicador: únicamente el $4.15 \%$ de la matrícula universitaria de aquel curso había participado en algún tipo de movilidad estudiantil y otras actividades en México y en el extranjero (Cedillo, 2012). Estas cifras evidencian que sigue siendo una minoría de alumnos los que realizan la movilidad, a pesar de las oportunidades que se ofrecen y del impulso político que recibe este propósito

El presente estudio se planteó explorar las posibilidades que ofrecen las redes sociales para favorecer la movilidad académica estudiantil. En específico nos planteamos averiguar el papel que han jugado las redes sociales en las experiencias de movilidad académica realizada por estudiantes de la Universidad de Colima, México.

\section{MÉTODO}

\subsection{Participantes}

De acuerdo con el objetivo establecido, la investigación focalizó a los estudiantes que hubieran realizado movilidad durante un semestre o un año en un mismo período de tiempo, por lo que se eligió el 2013 en virtud de que era el año inmediato anterior al curso académico en que se desarrolló este estudio. Con base en este criterio, la población base para seleccionar a los participantes fueron 319 alumnos que habían realizado movilidad de la Universidad de Colima en el año 2013, pertenecientes a 27 facultades y con 
experiencias en diversos destinos, en total 21 países, además de México. De ellos, 114 estudiantes aceptaron participar en este estudio.

Para un estudio más profundo y de carácter cualitativo se definió una submuestra de 17 personas. Para la selección de este subconjunto de participantes se siguió el criterio de intencionalidad con el fin de garantizar una representatividad suficiente de cada destino de la movilidad, entendidas como regiones: Europa (Francia, España, Italia, Países Bajos, Alemania, Dinamarca), Latinoamérica (Colombia, Cuba, Argentina, Perú, Chile y Brasil), Asia-Oceanía (Tailandia, Corea del Sur, Japón y China), Norteamérica (Estados Unidos y Canadá) y México (movilidad interna). Desafortunadamente, del grupo de Norteamérica y el grupo México no se recibieron respuestas de los estudiantes invitados. En resumen, la submuestra para esta segunda etapa del estudio quedó distribuida del modo siguiente: 7 estudiantes que habían realizado movilidad en universidades de Europa; 7 en Latinoamérica y 2 en la región de Asia-Oceanía.

\subsection{Técnicas y procedimiento}

En primer lugar se aplicó un cuestionario online de elaboración propia, validado para esta investigación, con base en las dimensiones propuestas por Haslberger (2005) relativas al proceso de ajuste a la comunidad receptora durante experiencias de transculturación. Este cuestionario incluyó 26 ítems distribuidos en seis bloques: (A) Perfil del alumno, (B) Movilidad, (C) Redes Sociales, (D) Adaptación transcultural, (E) Aprendizaje intercultural y (F) Emociones. La mayoría de las preguntas eran cerradas con opción de respuestas múltiples. En algunos bloques se incluyeron preguntas abiertas para que los estudiantes pudieran comentar aspectos generales sobre sus experiencias.

En un segundo momento se realizaron entrevistas grupales, las cuales permitieron profundizar en el estudio, así como contrastar la información con los resultados del cuestionario. La guía de preguntas para las entrevistas grupales se diseñó en concordancia con las del cuestionario, pero se formularon de manera más general, por lo que sólo fueron 10. No se consideró necesario indagar más acerca del perfil ni de otras variables sociodemográficas; tampoco sobre las emociones de manera explícita.

Por último y para efecto de contraste, también se realizó el análisis de contenido de muros del Facebook. Se realizó un análisis textual de la comunicación registrada en este espacio, directamente relacionada con las experiencias durante la movilidad académica. Para el análisis del contenido de las entrevistas y de los muros de Facebook se establecieron las temáticas, emergentes del proceso de análisis y consensuadas por las autoras de este artículo. Además, se segmentaron los discursos de las entrevistas y se 
clasificaron los contenidos de los muros de Facebook de mayor interés para responder al objetivo de la investigación. En este análisis se utilizó el software Maxqda para Windows.

\section{RESULTADOS}

El objetivo de esta investigación fue averiguar el papel que han jugado las redes sociales en las experiencias de movilidad académica realizada por estudiantes de la Universidad de Colima, México. A continuación se presentan de manera resumida los principales hallazgos, y siguiendo el orden del procedimiento utilizado para analizar los datos, primero se muestran los resultados de tipo cuantitativo basados en las respuestas al cuestionario. En seguida se insertan fragmentos de las entrevistas y de los contenidos de los muros de Facebook que ilustran, corroboran y/o amplían los resultados de los análisis cuantitativos. Los nombres de los alumnos que se muestran son ficticios, aunque el país citado sí corresponde al destino de la movilidad.

\section{Calidad de las interacciones durante la movilidad}

Respecto de las interacciones, entendidas como las relaciones con una persona local, pero en un momento negativo, se encontró que sólo 2 (4\%) participantes afirmaron haber pasado por esta situación. Esto puede ser entendido como que sería el grado más alto de integración, cuando puedes manejar una situación de esta naturaleza, por eso aparecen sólo esos registros. Casi sería un atrevimiento de un extranjero hacer algo así, aunque contara con la "compañía" de un local. En la Tabla 1 se muestran los resultados observados respecto a la calidad de las relaciones interpersonales.

\begin{tabular}{lcc}
\hline Orientación de la relación & Frecuencia & $\%$ \\
\hline Establecer lazos de amistad con la población local & 74 & 64.9 \\
Llegar a conocer a sus vecinos locales & 25 & 21.9 \\
Establecer contacto con los lugareños & 21 & 18.4 \\
Mantenimiento de relaciones con personas locales & 21 & 18.4 \\
Dar el primer paso para conocer a una persona local & 57 & 50 \\
\hline
\end{tabular}


El conocer y estar en contacto con una persona local fue fundamental para que los estudiantes entablaran relaciones con otras personas del lugar, tal y como lo muestra la Tabla 1.

La movilidad académica, como su nombre lo señala, tiene por objetivo el intercambio de saberes académicos, principalmente. Pero no es sólo eso, el alumno que vive esta experiencia está fuera de su cultura y establece contacto con compañeros de ésa y de otras culturas, que al igual que él están de intercambio en ese lugar. Esa interacción genera conocimientos tal y como muestran los datos de la Tabla 2. Estos resultados advierten de la importancia de las redes sociales para satisfacer esta necesidad.

\begin{tabular}{lcc}
\hline \multicolumn{1}{c}{ Motivos } & Frecuencia & $\%$ \\
\hline $\begin{array}{l}\text { Abolir estereotipos al aprender con el contacto } \\
\text { con personas de cultura diferente }\end{array}$ & 9 & 7.9 \\
$\begin{array}{l}\text { Conocimientos de distintas formas culturales } \\
\text { (tradiciones, costumbres, gastronomía, idioma, }\end{array}$ & 33 & 28.9 \\
etc.). & 22 & \\
Crecimiento personal con la interacción social & 20 & 19.3 \\
Aprendizaje social de otros lugares & 23 & 20.2 \\
Crecimiento profesional y personal & & \\
\hline
\end{tabular}

Tabla 2. Motivos asociados al interés por contactar con otras personas durante la movilidad N=114

¿Cuáles son las redes sociales que utilizaron los estudiantes y cómo apoyaron su proyecto de movilidad académica?

Facebook fue la red social que los estudiantes reportaron como más utilizada durante su experiencia de movilidad (95.6\%). Este porcentaje refleja la importancia y el potencial que tienen las redes sociales para este grupo etario dentro de todos los ámbitos. A continuación se muestran algunos usos que le dieron los jóvenes durante su experiencia de movilidad.

"(...) fue que por medio del Facebook conocí a las chicas con las que iba a vivir. Encontramos piso (gracias a) Facebook; encontramos a las compañeras. Los amigos con los que salíamos los conocimos por Facebook. Hay una organización en España, ESN creo, (...) y había un grupo de Facebook de todos los de intercambio en la ciudad. Para conocer gente o saber de fiestas y viajes, era por allí. Conocías gente, publicabas si querías ir al cine y ellos te acompañaban o te asesoraban sobre los sitios turísticos. Era la forma de comunicarnos allá (...)". [Alexis/ España] 
Los estudiantes refirieron que a través de Facebook contactaban con sus "caseros" antes de marchar al destino de su programa de movilidad, incluso manifestaron la creación de lazos afectivos que facilitaron la gestión y el acompañamiento durante esta experiencia:

"Y por medio de correo electrónico encontré casa. Ya tenía casa antes de llegar allá. (...) el señor con el que viví tenía como 70 años, pero usaba el Facebook (risas) yo lo veía (...) tenía cara de villano de (...) película antigua. Pero no, los señores eran un amor de personas, me tomaban casi como su nieto". [Michi/Colombia]

En otros países existen instituciones educativas que tienen grupos en las redes sociales con los estudiantes de movilidad en esa institución, específicamente en el Facebook, por lo que son integrados de forma automática; así se generan nuevas amistades que continúan después de concluir dicho proceso. A través del Facebook los estudiantes establecen vínculos con sus tutores, en ocasiones desde antes de irse, y se convierten en sus guías en la integración a esta comunidad.

“Llegando allá, la coordinadora de movilidad me agregó a un grupo para alumnos de movilidad, que éramos de 95 a 100. Y pues, que empiezan a agregar a los italianos, a los alemanes, a los españoles, mexicanos (que éramos un montón), los argentinos, los brasileños. Allí comenzaban a buscar casa, y la fiesta (donde los chilenos eran bien fiesteros)". [Michi/Colombia]

Facebook ha sido la red de apoyo a la comunicación de los estudiantes durante su movilidad académica; han recurrido a ella para satisfacer diferentes necesidades, aunque no parece que sea prioritario en el orden académico. Durante las entrevistas se detectó que también se utilizaron las oportunidades comunicativas que ofrecen otras herramientas como Skype y Whatsapp, las cuales también permiten la creación de "grupos" del tipo redes. Al parecer, los jóvenes estudiantes unen de manera casi automática el concepto de redes sociales a Facebook, porque en el cuestionario sólo apareció una mención a Twiter, otra a Instragram y una persona marcó la opción "combinación de varias".

A través de las entrevistas, los estudiantes fueron claros respecto a que hacen un uso diferenciado de las redes; los estudiantes optan por usar Skype como una alternativa para comunicarse con las personas más cercanas ya que permite "verse".

Los resultados que se muestran en la Figura 1 evidencian que tanto la comunicación como el acompañamiento de la familia y amigos en las redes sociales ofrecieron a los estudiantes un significativo apoyo: el $82.5 \%$ de los encuestados lo afirmó cuando se les 
preguntó sobre la funcionalidad atribuida a las redes sociales durante su experiencia de movilidad académica.

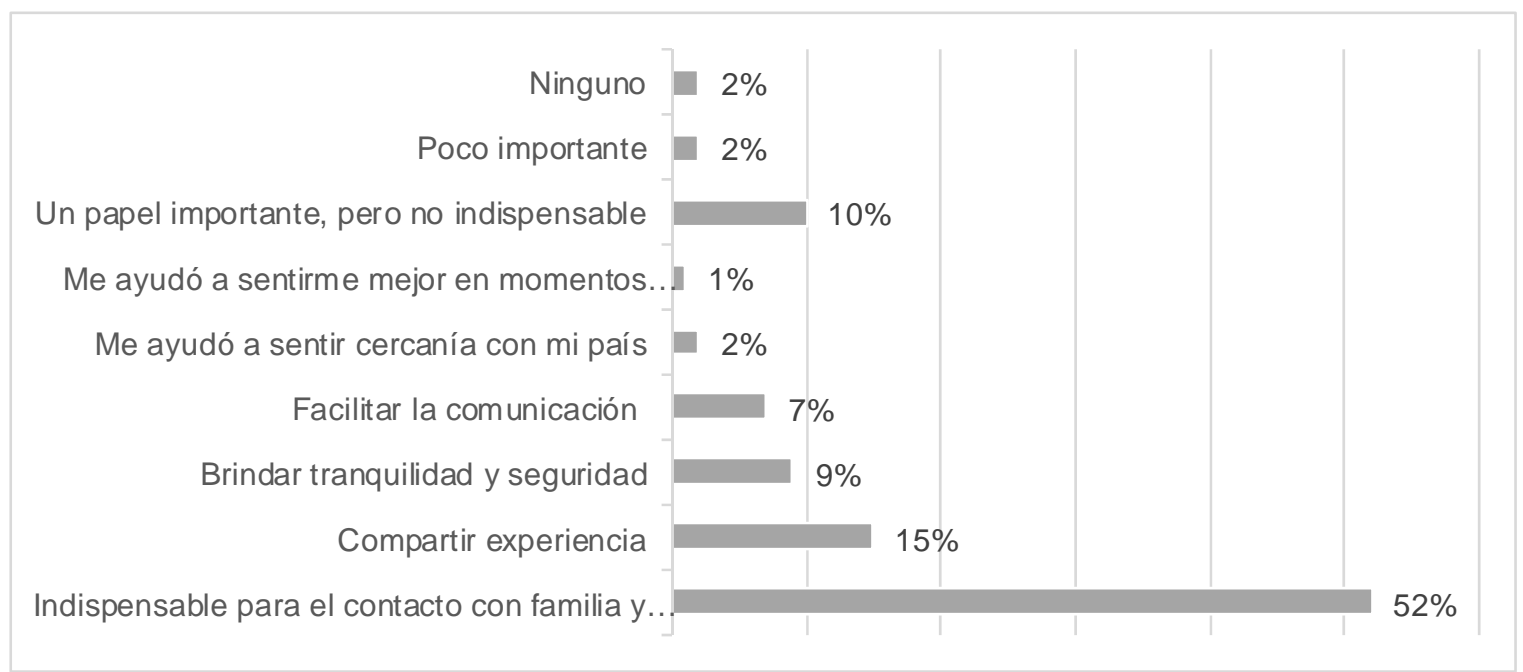

Figura 1. Importancia y funciones atribuidas a las redes sociales durante la movilidad académica.

Esta información se corroboró también en el análisis de las entrevistas, como se muestra en el fragmento siguiente:

"Sí, platicábamos de todo, me enteraba de todo. (...) en realidad yo no me sentía lejos por el contacto que tenía con mi familia, con mis amigos, y no porque me la pasaba hablando con ellos, sino porque veía lo que estaban publicando en Facebook. Eso me ayudó en todo momento a no resentirme". [Cynthia/ España]

En los relatos anteriores también se aprecia que la comunicación a través de las redes evidencia fundamentalmente los sentimientos positivos experimentados durante la movilidad. En este sentido, los datos recopilados a través de la encuesta lo patentizan (ver Figura 2). 


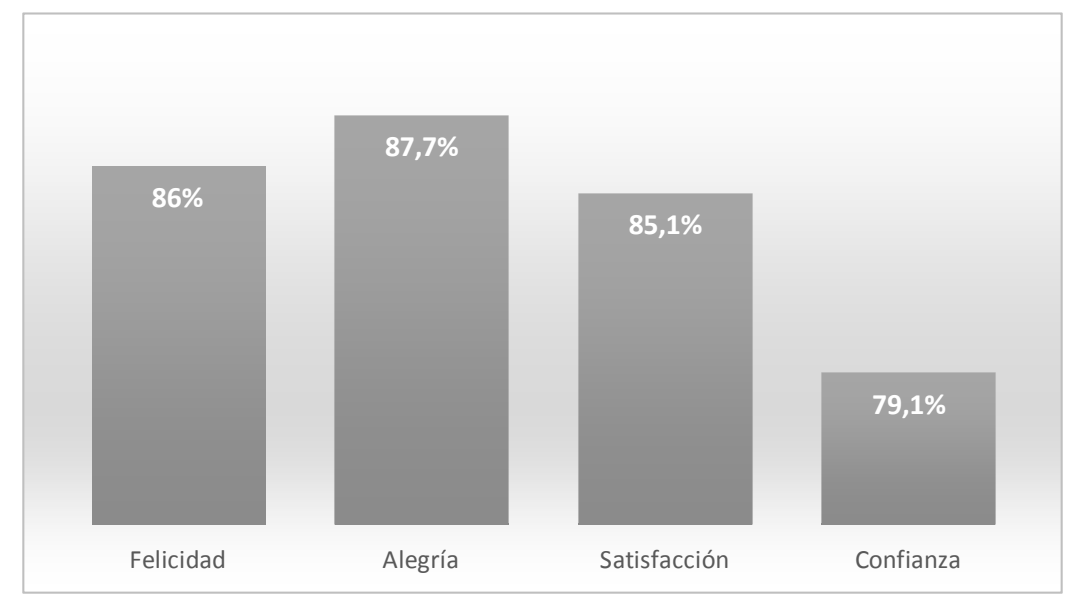

Figura 2. Sentimientos positivos relacionados con la experiencia de movilidad.

En cambio, las referencias a emociones negativas fueron poco frecuentes: ansiedad $(9.6 \%)$, temor $(9.6 \%)$, impaciencia $(6.1 \%)$, tristeza $(6.1 \%)$, aburrimiento $(5.3 \%)$ y decepción $(2.6 \%)$, ira (2.6\%).

"Al principio, con los que vivía eran mexicanos, pero no eran mis amigos: vivía con ellos porque todos íbamos para Málaga. No conocía a nadie, no tenía la confianza $100 \%$ de platicar y me conectaba a Skype. Mi papá todo el tiempo me hablaba, me marcaba (...).Yo creo que nada más fue la primer semana en la que me sentí sola y abandonada porque ya después nunca estaba en la computadora atenta a Skype. Por Whatsapp "Hola pa' sigo bien" "Hola ma estoy bien", una foto de "sigo completa". No era tanto el estar hablando seguido, sino de vez en cuando". [Samantha/ España]

La voluntad de integrar las experiencias de la movilidad a la dinámica de las redes sociales que manejan habitualmente fue un hecho ampliamente constatado:

"Había un grupo de FB en el que vendían cosas usadas en Alborg. (...) yo me acuerdo que compraba cosas muy baratas, de100 coronas o algo así, cuando en las tiendas costaba 500, porque lo publicaban en Facebook (...). Regalan muebles y suben las fotos (...) ponen que lo regalan al que llegue primero. Era un grupo en el que todo el mundo estaba, todos los estudiantes internacionales, hasta los daneses, y siempre había algo barato". [Karla/Dinamarca]. 


\section{DISCUSIÓN Y CONCLUSIONES}

Este estudio confirma que las redes sociales son el espacio de comunicación del cual forman parte los jóvenes, como han demostrado diversos estudios realizados en Estados Unidos (Ellison et al. 2007, Lenhart y Madden, 2007; Miller et al. 2010). Los estudiantes de la Universidad de Colima también están integrados a diversas redes sociales y las utilizan en los diferentes procesos en los que participaron durante su experiencia de movilidad. Este resultado afianza el supuesto del amplio potencial socio-educativo de las resdes sociales (Alvarez y Olivera-Smith, 2013, Rodera, 2011),

Al parecer, la movilidad académica incentiva la creación de grupos en las redes sociales, específicamente a través de Facebook, Este escenario comunicativo promueve y facilita, básicamente, el establecimiento de vínculos entre sus participantes (Haythornthwaite, 2002). En este sentido, nuestro estudio patentiza el destacado papel de las redes sociales en los procesos de integración grupal y acompañamiento emocional, hecho que puede favorecer el ajuste sociocultural del comportamiento durante las trayectorias internacionales de los estudiantes (Coles y Swami, 2012).

Nuestro estudio también constató que el contenido de la comunicación que tiene lugar en estos espacios posee carácter informal, parte de la comunión de intereses (Boyd y Ellison, 2007) y evidencia una funcionalidad orientada a la búsqueda y a la expresión de apoyo instrumental y afectivo (Belli y De Eugenio, 2014; Madgea et al. 2009).

Los estudiantes que optan por la movilidad utilizan las redes sociales como un recurso para sentirse acompañados; un acompañamiento que lo informa, que lo acoge en su nueva comunidad, que lo integra a otros grupos y que al mismo tiempo lo mantiene como integrante de sus grupos anteriores. Y esa integración, tanto con referencia a la nueva comunidad, como a seguir integrado a la que pertenece, se encuentra inevitablemente unida a las cuestiones de identidad, como han constatado otros estudios en esta línea (Aguilar, y Said, 2010, Quintana y Valbuena, 2015). Emerge una identidad en construcción off/online, igual que sus vidas que transcurren en estas dos vías.

Sin embargo, aun no encontramos signos importantes sobre el uso de este escenario como apoyo a actividades académicas. Este sigue siendo un asunto pendiente, quizá conviene introducirlo en las agendas institucionales, a fin de canalizar diversos apoyos, como sugiere Castell (2004).

En definitiva, la comunidad académica también debe estar presente en ese círculo con el alumno que se va de movilidad, quien debe sentir que está presente en su lugar de origen, 
pero desde la academia, no sólo a través de sus compañeros. Al menos estas son las expectativas de los estudiantes, como queda reflejado a través del uso del Facebook.

Aunque modestos y limitados por la representatividad de la muestra de participantes, consideramos que estos hallazgos pueden ser un referente valioso en este sentido, en particular para la Universidad de Colima, escenario motivador de esta investigación. En última instancia, interesa encontrar alternativas aceptables y prácticas para impulsar su utilización como un apoyo añadido y esencial para los estudiantes que participan de los programas de movilidad académica internacional.

\section{Agradecimientos}

La investigación que reporta este artículo es parte del proyecto de investigación el "Papel de las redes sociales por internet, en el ajuste sociocultural en las comunidades receptoras de los estudiantes de movilidad de la Universidad de Colima", realizada con el soporte de la Universidad de Colima, México, Convocatoria FRABA 14-2013. Referencia del proyecto: FRABA_827-13. 


\section{REFERENCIAS}

AGUILAR RODRÍGUEZ, D. E., y SAID HUNG, E. (2010). Identidad y subjetividad en las redes sociales virtuales: caso de Facebook. Zona próxima, 12, 190-207.

ALVAREZ, I. M., \& OLIVERA-SMITH, M. (2013). Learning in social networks: rationale and ideas for its implementation in higher education. Education Sciences,3, 314-325.

ARNOLD, N., \& PAULUS, T. (2010). Using a social networking site for experiential learning: Appropriating, lurking, modeling and community building. Internet and Higher Education, 13, 188-196.

AMIPICI (Asociación Mexicana de Internet) (2014). Estudio sobre los hábitos de los usuarios de Internet en México. Recuperado el 26 de marzo de 2015 de https://www.amipci.org.mx/estudios/habitos_de_internet/Estudio_Habitos_del_Int ernauta_Mexicano_2014_V_MD.pdf

BELLI, S., \& De EUGENIO, G. (2014). Prácticas emocionales y procesos subjetivadores en la sociedad digital: el caso de los suicidios online. Quaderns, 16(2), 57-72.

BOYD, D.M.; ELLISON, N.B. (2007). Social network sites: Definition, history, and scholarship. Journal of Computer-Mediated Communication, 13, 210-230.

BROVETTO, J. (2008). Espacio común de la educación superior en América Latina y el Caribe. Educación Superior y Sociedad, (13), 116-124.

CASTELLS, M. (2004). The networksociety: A cross-cultural Perspective. Northampton: Edward Edgar.

CEDILlO, R. R. (2012). Informe de Labores 2012. Universidad de Colima. México. [documento en línea] Recuperado el 26 de marzo de 2015 de http://www.ucol.mx/transparencia/docs/informes\%20rector/Informe2012\%20recto ria.pdf

COLES, R. \& SWAMI, V. (2012).The sociocultural adjustment trajectory of international university students and the role of university structures: A qualitative investigation. Journal of Research in International Education, 87-100. 
DE WIT, H. (2011). Globalización e internacionalización de la educación superior [introducción a monográfico en línea]. Revista de Universidad y Sociedad del Conocimiento (RUSC), 8 (2), págs. 77-84. UOC. Recuperado el 6 de mayo de 2015 de http://rusc.uoc.edu/ojs/index.php/rusc/article/view/v8n2-dewit/v8n2-dewit

ERTMER, P.A., \& STEPICH, D.A. (2004). Examining the relationship between higher-order learning and students' perceived sense of community in an online learning environment. En Proceedings of the 10th Australian World Wide Web Conference, Gold Coast, Australia, 3-7 July 2004.

HASLBERGER, A. (2005). Facets and dimensions of cross-cultural adaptation: refining tools. Ashridge Business School UK. Emerald Research Register. Recuperado el 6 de mayo de 2015 de www.emeraldinsight.com/0048-3486.htm

HAUG, G. (2010). La internacionalización de la educación superior: más allá de la movilidad europea. La Cuestión Universitaria, 6, 20-29. [Boletín electrónico de la Cátedra Unesco de Gestión y Política Universitaria de la Universidad Politécnica de Madrid]. Recuperado el 6 de mayo de 2015 de http://www.lacuestionuniversitaria.upm.es/web/grafica/articulos/imgs_boletin_6/p dfs/LCU-6-3.pdf

HAYTHORNTHWAITE, C. (2002). Building social networks via computer networks: creating and sustaining distributed learning communities. En Renninger, K.A., Shumar, W., (Eds). Building Virtual Communities: Learning and Change in Cyberspace (pp. 159190). Cambridge University Press: Cambridge, UK

LENHART, A., \& Madden, M. (2007). Teens, privacy \& online social networks: How teens manage their online identities and personal information in the age of MySpace (Report). Pew Internet \& American Life Project. Recuperado el 7 de mayo de 2015 de http://www.pewinternet.org/2007/04/18/teens-privacy-and-online-socialnetworks/

MADGE, C., MEEK, J., WELLENS, J., \& HOOLEY, T. (2009). Facebook, social integration and informal learning at university: 'It is more for socialising and talking to friends about work than for actually doing work'. Learning, Media and Technology, 34, 141-155.

MARMOLEJO, F. (2012). Trends in international mobility of students: a Wake-Up Call for the U.S.? The Chronicle of Higher Education. Recuperado el 7 de mayo de 2015 de http://chronicle.com/blogs/worldwise/education-at-a-glance-2012/30454 
MARTÍNEZ, E. y LAZO, C. M. (2011). Jóvenes interactivos: Nuevos modos de comunicarse. Oleieros (La Coruña): Gesbiblo.

MILLER, R., PARSONS, K., \& LIFER, D. (2010). Students and social networking sites: the posting paradox. Behavior and Information Technology, 29, 377-382.

PALLOFF, R.M., \& PRATT, K. (1999). Building Learning Communities in Cyberspace. San Francisco CA: Jossey-Bass.

QUINTANA, M. F., \& VALBUENA, W. (2015). Cibercomunicación: ¿necesidad o satisfactor? Análisis de facebook desde las necesidades axiológicas.Civilizar Ciencias de la Comunicación, 1(1), 59-74.

RODERA, A. M. (2011). El proyecto Facebook y la post-universidad. Sistemas operativos sociales y entornos abiertos de aprendizaje [reseña en línea]. RUSC Revista de Universidad y Sociedad del Conocimiento, 8 (2),165-169. UOC. Recuperado el 7de mayo de 2015 de http://rusc.uoc.edu/ojs/index.php/rusc/article/view/v8n2rodera/v8n2-rodera

TÚÑEZ LÓPEZ, M. y GARCÍA, J. S. (2012). Las redes sociales como entorno docente: análisis del uso de Facebook en la docencia universitaria. Píxel-Bit: Revista de medios y educación 41, 77-92.

UNESCO (2009). Tendencias mundiales en materia de movilidad estudiantil [Boletín EDUINFO, junio- julio 09]. Recuperado el 7 de Mayo de 2015 de http://portal.unesco.org/education/es/ev.php-

URL ID=59341\&URL DO=DO TOPIC\&URL SECTION=201.html

\section{Para citar este artículo:}

Rocha, M. A.; Alvarez, I. M. \& Amador, G. (2015). Las redes sociales: el acompañamiento emocional de los estudiantes durante la movilidad estudiantil universitaria. EDUTEC, Revista Electrónica de Tecnología Educativa, 53. Recuperado el dd/mm/aa de http://www.edutec.es/revista 
